\title{
ON THE REAL-WORLD-SEMANTICS INTERPRETABILITY OF FUZZY RULE BASED SYSTEMS UNDER FUZZY SET APPROACH AND HEDGE ALGEBRA APPROACH
}

\author{
THU ANH NGUYEN ${ }^{1}$, CAT HO NGUYEN ${ }^{1,2}$ \\ ${ }^{1}$ Institute of Information Technology, Vietnam Academy of Science and Technology \\ ${ }^{2}$ Institute of Basic Research and Application, Duy Tan University at Da Nang \\ ${ }^{1}$ thuanh@ioit.ac.vn; ${ }^{2}$ ncatho@gmail.com
}

\begin{abstract}
The study will further discuss the novel real-world-semantics-based approach (RWSapproach) to the interpretability of fuzzy systems proposed in [8] to show that the RWS-interpretability of fuzzy systems in this approach is very essential and practical. It is also analyzed that the usual theories as in mathematics and physics are all RWS-interpretable or, roughly speaking, they are able to model their real-world parts, properly. It is pointed out that though the fuzzy set theory is a great one which is very flexible, has many advantages in the application and obtains numerous achievements, methodologically, it still has an essential shortcoming that it is not RWS-interpretable on the viewpoint of this RWS-approach. To ensure the RWS-interpretability of a fuzzy linguistic system, it is argued that word-domains of variables with their own order-based semantics should be made use and interpreted as a formal bridge to connect the real-world semantics with the constructed fuzzy linguistic system that works on the designed computational semantics of linguistic words. It is initially shown that there exists a formalism based on the theory of hedge algebras to design RWS-interpretable fuzzy systems.
\end{abstract}

Keywords. Real-world-semantics-based approach, real-world-semantics-based interpretability, linguistic fuzzy rule bases, fuzzy system.

\section{INTRODUCTION}

It is obvious that real world (RW-) owns numerous various structures and humans cognize the RW-structures based on their formal languages including formal theories in distinct fields such as mathematics, physics, chemistry, social-economy..., which are developed based on their own formalisms. Of course, the ultimate aim of these formal theories is to apply them to develop techniques and methods to handle formal objects, which semantically express their corresponding real-world entities, in terms of the formalisms of the theories to solve realworld problems. To guarantee the effect of such developed methods, it is implicitly required in practice that these theories should be developed in a strong relation with the structures of the theory real-world counterparts. For illustration, let us consider a very simple theory, the theory of real numbers, to serve for calculating the length of segments of straight lines in reality. The theory is developed relying on a formal language using decimal (symbolic) strings in an axiomatic way based on well-known axioms which represent specific key relationships between the lengths of line segments discovered in the real-world counterparts. This theory 
developed in such a way to ensure that it has a close relation with the real-world counterparts which are the straight lines on the earth under assumption that they are actually straight.

While the math- and physical theories, for instance, are developed in close relations with reality, many theories/methods in the fuzzy set framework are developed based on very weak formal relationships with their real-world counterparts. As an example, let us consider the symbolic approach to fuzzy multi-criteria group decision making problems in a fuzzy linguistic environment. In such an approach, its fundamental structure is linguistic assessment scales of the form $S=\left\{l_{0}, \ldots, l_{n}\right\}$ used instead of numerical assessment ones, where $l_{i}^{\prime}$ s are linguistic labels whose semantics induces a linear order that satisfies the condition that $l_{i}<l_{j}$ iff $i<j$ [4]. In general, $S$ can be considered as a subset of the worddomain of a linguistic variable $X$, e.g. $X$ is the variable "technology" of an equipment bid. Since $S$ is still not interpreted as a math-structure in the fuzzy set framework and it has no numerically computational features, instead to compute on the linguistic assessments $l_{i}^{\prime} \mathrm{s}$, one has to establish a computational formalism to compute on their respective indexes without any justification that these indexes express the real-world semantics of $l_{i}^{\prime}$ s. The numeric result of such a computation, denoted by $r$, is translated into the linguistic label whose index is the round number of $r$ without an explanation why one can manage linguistic labels in such a manner. This approach and its extended ones have attracted many studies in almost last two decades, but it can be observed that there are no logical and mathematical bases to support this computational formalism $[4,11,12,2]$. On our study's standpoint, this situation is an inevitable consequence of the lack of a formal connection of the indexes of linguistic labels of $S$ with their real-world semantics. Similarly, to simulate human capabilities in handing linguistic words in the framework of fuzzy sets, it is assumed that the fuzzy sets under consideration are always associated with their linguistic words assigned intuitively by user. However, the operations, say union or intersection, on the fuzzy sets are also defined without any explicit relation with the respective operations OR or AND on their assigned words to guarantee that the behavior of the union or the intersection on the fuzzy sets is appropriate to the one of the OR or the AND acting on their words. Noting that the operations on fuzzy sets are always defined in the set of all fuzzy sets of an uncountable universe $U$, while the set of linguistic words is only finite, we can follow that the behavior of the operations on fuzzy sets is not appropriate to the one of the respective operation of their assigned words as it will be discussed in sequels.

This motivates us to state that the functionality of every natural language can be strongly related with the RW, i.e. the semantics of their elements express certain entities/relationships in the RW, and hence they may be utilized to form a bridge to connect the fuzzy sets and the operation on them with the real-world semantics (RWS) of the corresponding operations acting on their assigned words. For this reason, the study [8] proposed a novel RWS-approach to the interpretability of the fuzzy sets and introduced a new concept of the RWS-interpretability of the fuzzy system (FSyst) components, to guarantee that the semantics of their components must represent a real-world semantics or they must specify their entities in the RW. The ultimate purpose of the approach is to establish a methodology for designing FSysts to ensure that the behavior of the designed FSysts will meet the designer expectation.

Following the novel RWS-approach to the FSyst interpretability proposed in [8], in this study we will examine the following main problems: 
- To make the RWS-interpretability concept more understandable and to further justify the RWS-interpretability of natural human languages and word-domains of variables and its elementary role in examination of the RWS-interpretability of the FSyst components.

- To examine the RWS-interpretability of basic operations of the fuzzy set theory which is oriented for the first time to answer the question whether this theory is RWSinterpretable.

- To show an ability to solve the RWS-interpretability of some approximate reasoning method based on the theory of hedge algebras (HAs) and their quantification.

The paper is organized as follows. In Section 2, a short overview on the introduction of the RWS-approach to the FSyst interpretability and a general discussion on the RWS-interpretability of non-fuzzy and fuzzy theories and methods, specially, on the RWSinterpretability of HAs. Section 3 is devoted to an examination of the RWS-interpretability of FSyst components which is considered as a further study [8]. Section 4 presents some first results in studying the RWS-interpretability of the fuzzy set theory and of HAs. Some conclusions are given in Section 5.

\section{RWS-INTERPRETABILITY OF VARIABLE WORD-DOMAINS \& ITS CRUCIAL ROLE IN STUDYING THE RWS-INTERPRETABILITY OF FUZZY THEORIES}

In mathematics and physics there are various formal theories that are developed in axiomatic manners and they are RWS-interpretable because their axioms represent certain essential properties of their research RW-counterpart in terms of their own formalism. This statement is justified by numerous achievements of the applications of these theories to solve social or technological problems to successfully develop the societies. However, as it will be seen below, there are many problems for the theories dealing with fuzzy linguistic data as, methodologically, they lack necessary formal bases to connect to the RW. This situation motivates the authors of [8] to introduce and initially examine the RWS-approach to the interpretability of FSysts to establish a methodological foundation to ensure that the behavior of the FSysts designed based on this foundation in reality is just expected by the designers.

\subsection{The novel concept of RWS-interpretability of any formalized theories -A deeper discussion}

This concept was proposed and initially examined in [8], but due to the space limit of its publication it is difficult to present such a complex and novel concept more comprehensively. In this study, we will more explicitly and deeply analyze practical and methodological necessity of the study of the RW-interpretability of formalized theories, in general, and of formalized FSysts, in particular, where the terminology "formalized" used in this study is to emphasize that it should be careful to deal with the relationship between symbolic expressions viewed on the syntactical standpoint and their semantics which is interpreted in a computational structure. This is very essential because, methodologically, human beings cognize the reality around their daily lives by using symbolic languages associated with implicit 
semantic interpretation assignments, by which their elementary symbolic elements convey real-world semantics, such as natural languages of human communities, mathematical languages, physical languages... So, it is necessary to put the study of the FSyst interpretability in relationships between human beings, the $R W$ and natural languages, as it is exhibited by a scheme given in Fig. 1. Remember that every natural language has been taking form by human beings when they interact with their RW in a very long period of the human development history. This is the practical basis to ensure the RW-interpretability of the natural languages.

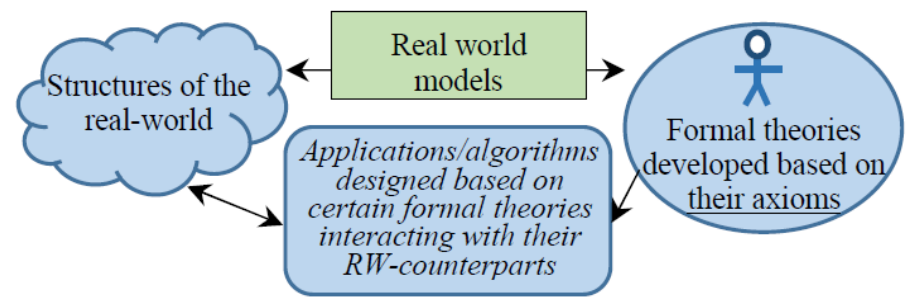

Figure 1. Relationships between formalized theories, their models and applications and their RW counterparts

To make this new RW-interpretability concept easy to understand, let us first consider simpler theories in the fields of computer science or mathematics. It is useful to recall ourselves the crucially important role of mathematics, one feature of which saying that "The core knowledge of computer science is nothing but mathematics" and, in addition, it is observed that the semantics of the elements (represented by symbolic expressions) of the underlying set of any given math-theory Tare nothing but their relationships in its structure developed on its underlying set. When a computer handles symbolic expressions represented in the computer to simulate a process in reality in a right way, it is required that the computational semantics of these expressions handled by the computer in a certain math-formalism must rightly express their RW-meaning, i.e. certain RW-objects assigned to them, which in nature is completely different from the computational semantics. Since relationships between elements are essential, similarly as in the field of the mathematics, one way to model the RW-semantics of RW-objects is to establish an interpretation mapping from the RW-objects to their computational ones so that this mapping can preserve the discovered relationships between the RW-objects in question.

From this analysis, the RWS-approach to the FSyst interpretability proposed in [8] is actually examined under the following assumptions that are explicitly formulated in this study as follows:

- The RW and its parts always have their own structures, which are collections of relationships between their RW-entities/objects.

- The natural languages of human communities are RWS-interpretable, i.e. their elements used in each other's communication and in decision making can properly convey RW-semantics. This point will be discussed in more details next.

- Since the ultimate purpose of the fuzzy sets theory is to simulate human capacities 
in handling fuzzy linguistic words, every fuzzy set under consideration of a linguistic variable must always be associated with a fuzzy linguistic word.

- Since in this approach, RW plays a basic role in studying the interpretability not only of the FSysts, but also of any formalized theories, an RW-structure can be modeled by a formalized fuzzy linguistic theory $T_{L}$ as well as by a formalized numeric theory $T_{N}$. For this reason, if $x$ denotes a variable in the RW, then $x_{L}\left(x_{N}\right)$ denotes the same variable $x$ modelled by $T_{L}\left(T_{N}\right)$. It will be seen that this notation is useful in the examination of the RWS-interpretability of FSysts to easily present the semantic compatibility between two theories $T_{L}$ and $T_{N}$ when they model the same part of the RW to solve a problem.

\subsubsection{The concept of RWS-interpretability of formalized theories}

A FSyst whose functionality is to produce outputs from given input data and its fuzzy knowledge can be considered as a formal theory $T_{F S y t}$. As discussed above and from the above given assumptions, for a given FSyst we denote by $S_{R W P}$ the structure of a Real World Part (RWP), that represent relationships of its RW-counterpart objects of the theory $T_{F S y t} . S_{R W P}$ describes relationships between RW-variables in the $S_{R W P}$. Roughly speaking, in the RWS-approach, to ensure that the theory $T_{F S y t}$ can model the structure $S_{R W P}$ properly, one has to establish an interpretation mapping $f$ from the objects of RW-variables to computational objects of the corresponding variables of $T_{F S y t}$ so that $f$ does preserve key observed essential relationships within $S_{R W P}$. This idea suggests the authors of [8] to apply the concept of the interpretability of a theory $S$ in another one $T$ defined by Tarski et al. in [10] which can be formulated informally as: assuming $T$ and $S$ are formal theories, $S$ is said to be interpretable in $T$ if there is an interpretation mapping from the formalized language underlying $S$ into the formalized language underlying $T$ so that every theorem of $S$ can be translated into and proved in $T$. That is, the interpretation mapping can convey the structure of $S$ into the one of $T$. In mathematics, it is necessary to required that this mapping can convey the axioms (i.e. key relationships) of $S$ into certain theorems of $T$. Therefore, the study [8] introduces the following definition.

Definition 1. ([8]) A formalized method/theory $T$ formulated in its formalized language to simulate a real-world structure, denoted by $W_{T}$, is said to be RWS-interpretable if there exists an interpretation mapping $R_{T}: W_{T} \rightarrow T$, which assigns real-world objects of $W$ to elementary formalized elements of $T$ that can convey or preserve the essential properties of $W_{T}$. In this case, $T$ is called an RWS-model of $W_{T}$ or $W_{T}$ is interpretable in $T$. Such a formalized method $T$ is called $R W S$-interpretable. Note that, the structure $W_{T}$ is a subjective concept as it depends on the observation/perception of a human user. In this sense, most of classical mathematical theories are RWS-interpretable.

Example 1. The classical math-theories, like the theory of the real numbers, math-analysis, the Cantor's set theory, metric spaces..., are RWS-interpretable. It can easily be verified that the operations and axioms of these theories represent relationships of RW-objects in their corresponding parts of the RW discovered by human founders whereby readers/students can understand the theories.

Example 2. If the RWS-interpretability of the theories in Example 1 looks like at first 
glance natural, but, as it will be discussed in the next section, that the fuzzy sets theory seems to be not RWS-interpretable and, thus, the RWS-interpretability of fuzzy linguistic theories seems to be a quite novel problem. A question that arises is that does there exist such a theory which is RWS-interpretable. Therefore, this example aims to argue that the theory of the HAs immediately dealing with fuzzy linguistic words is RWS-interpretable under a previously adopted assumption that human community natural languages are RWSinterpretable. This question will be proved in sequel and, here, we only give some facts supporting this assertion.

Since natural languages are RWS-interpretable, the word-domains of variables that can be recognized as order-based structures induced by the inherent order-based semantics of their words are also RWS-interpretable. For example, assume firstly that for every RW-variable $x$, $x_{L}$ and $x_{N}$ are, respectively, the linguistic variable with linguistic domain $\operatorname{LDom}\left(x_{L}\right)$ and the numeric variable with numeric domain $N \operatorname{Dom}\left(x_{N}\right)$ used to describe the variation of $x$ in the RW. It is observed that if the first state of the RW-variable $x$ is smaller than its second one, then so are the two respective descriptions of either $x_{L}$ or $x_{N}$. That is, the order relation of two linguistic words of $\operatorname{LDom}\left(x_{L}\right)$ expressing the RW-relationship between the two (RW-) states of $x$ observed by a human user and expressed in terms of his words is compatible with the respective numeric states of $x_{N}$ observed by the same user. Similar as for the theory of the real numbers, since the axioms of a HA represent certain key essential relationships between words of the word-domain under consideration and the HAs is developed in an axiomatic manner, this ensures the RWS-interpretability of the HAs.

Based on the concept of the interpretability defined in Def. 1 and the successful applications of math-theories in reality, we adopt the following hypothesis.

Hypothesis 1. The developments of math-theories based on axiomatic methods ensure their $R W S$-interpretability, that is, a math-theory with its axioms whose semantics is justified to represent key structural relationships between entities of the RW-counterpart of the theory is $R W S$-interpretable.

\subsubsection{Proposal of a scheme to solve a RWS-interpretablity problem}

In math-logics, the inference mechanisms of predicate logics guarantee that a conclusion derived from valid statements is also valid. However, as RW-structures are not formalized theories, according to our knowledge, the above hypothesis could not be proved even in the field of mathematics. In the fuzzy/uncertain environment with inexact statements, one has no strict mechanism that permits to derive valid statements from given valid ones, a similar assertion is even more difficult to prove. In addition, as discussed in [8], the RWS-interpretability concept is subjective as it depends on which RW-features of the RW-counterpart the developer can discover, especially when one deals with problems in a fuzzy environment. In such complex environment which may even involve several distinct structures in the same RW-counterpart, one may not observe all necessary RW-features to solve a given problem. For instance, the generality-specificity of words has not been considered in the fuzzy set framework. Thus, it is necessary to introduce a scheme to solve a given RWS-interpretability problem as shown in Fig. 2, where the RWS-interpretability of a formalized fuzzy expression depends on which a structure of its RW-counterpart can be discovered, including expressions representing Approximate Reasoning Methods (ARMs). A 
given component of a FSyst or even the whole FSyst itself, or any method/algorithm can be considered a formalized expression $E$ that is formulated to solve a problem in a part of the RW, $\mathcal{W}$, with its own RW-structure $S_{W}$. For example, for a given fuzzy rule base $F R B$ formulated by a human expert to solve a problem discovered by him in a certain part of the RW, assume that its rules are understood as being combined by a logical conjunction. Let $L R B$ be obtained from FRB by replacing the occurrences of fuzzy sets in FRB with their respective associated linguistic words. Then, $L E$ is the linguistic expression obtained by the conjunction of the linguistic rules of $L R B$. Of course, to solve the discovered problem, one has to translate $L E$ into a certain computational expression, $C E$, of a computational space constructed by the human expert, $C S$, whose elementary elements must represent the semantics of words used by him to describe the basic objects the RWP $\mathcal{W}$.

\begin{tabular}{|c|c|c|}
\hline $\begin{array}{l}\text { Given } W \text { with its } \\
\text { own structure } S_{W} \\
\text { and } L E \text { formulated } \\
\text { by an expert, try to } \\
\text { discover key } \\
\text { relationships } \\
\text { between variables } \\
\text { of } S_{W} \text { and between } \\
\text { elements of the } \\
\text { same variable }\end{array}$ & $\begin{array}{l}\text { (i) Build a compu- } \\
\text { tational space } C S_{W} \\
\text { (ii) Define neces- } \\
\text { sary notions and } \\
\text { key relationships } \\
\text { in } C S_{W} \text { that can } \\
\text { model the respe- } \\
\text { ctive ones dis- } \\
\text { covered in } S_{W}\end{array}$ & $\begin{array}{l}+ \text { Build a } \\
\text { mapping } f \text { to } \\
\text { translate } S_{W} \\
\text { into } C S_{W} \text {. } \\
+ \text { Check } \\
\text { whether } f \text { can } \\
\text { maintain the } \\
\text { key relation- } \\
\text { ships of } S_{W}\end{array}$ \\
\hline
\end{tabular}

Figure 2. Scheme to solve an RWS-interpretability problem

So, in the RWS-approach it is required that the $C E$ must be RWS-interpretable when representing the RW-semantics of $L E$ in $\mathcal{W}$. The scheme shown in Fig. 2 comprises the following main tasks:

Task 1. For a given $\mathcal{W}$ and the linguistic expression $L E$ formulated by a human expert, it is necessary to discover all necessary key features of the structure $S_{W}$ of $\mathcal{W}$, noting that $S_{W}$ may contain several substructures that are distinct in nature. For example, the order-based relationships and the generality-specificity relationships define quite different structures between the words of the same linguistic variable. Based on these discovered features, one should formulate constraints that impose on the interpretation mapping defined in Task 3.

Task 2. To make use of certain computational formalism, construct numerically computational space $C S_{W}$ which is appropriate to solve the problem arising in the real-world part $W$. Noting that the objects of $S_{W}$ are completely different in nature from the corresponding ones of $C S_{W}$, to model the discovered structure $S_{W}$, one should define suitable notions and necessary relationships in $C S_{W}$ that serve for the representation of key characteristics of the structure $S_{W}$ in the computational structure $C S_{W}$.

Task 3. Build an interpretation mapping $f$ from the RW-objects to computational objects of $C S_{W}$ so that $f$ is satisfied by the constraints formulated in Task 1 to convey key features of $S_{W}$ to the corresponding ones in $C S_{W}$.

In sequel, we will demonstrate important and useful role of this scheme in examining the RWS-interpretability of the components of FSyst. 


\subsection{The RWS-interpretability of human natural languages and of HAs of vari- ables}

Since the concept of RWS-interpretability of a formalized theory/expression is very essential, but it is a pity that theories and methods in the fuzzy linguistic environment, in general, are not RWS-interpretable. One of main reason is that they have no formal basis to connect with natural languages which are RWS-interpretable, as we will argue in the following point.

\subsubsection{The RWS-interpretability of natural languages of any human communi- ties}

This point has still not explicitly discussed in the study [8], however, it is methodologically very essential and, hence, it is necessary to discuss about it here.

Every language of a human community considered as a whole cannot be formalized as a structure and, therefore, we cannot show that it is RWS-interpretable in the sense of Def. 1. However, the history of fighting for the human existence and development, in which its language has been being used throughout the length of its history to communicate with each other or to make decisions in daily lives, proves that its language must be RWS-interpretable, otherwise the human community cannot exist.

As a consequence, the word-domain of a variable, i.e. the set of all words in the language that are the human community uses to describe linguistically the states (or, values) of the variable. Moreover, it can be seen that every word-domain of the language is, at least, partially ordered based on the meaning of its words. It is more essential that this semantic order relation on this word-domain has been taken shape in the long-term history of the community to serve for daily life decision making, for which the comparison of values of decision criteria to choose a better alternative among other ones is crucial. The successes of human beings in their activities of decision making result in a consequence that the comparison between alternatives of a decision making problem based on the order-based relationships of the words used to describe the one decision alternatives are appropriate to the reality. That is, natural languages provide actually powerful functionality to be used to describe all things in the RW serving for human cognition and decision making. In other words, we have

Hypothesis 2. Any human natural languages are RWS-interpretable.

The RWS-interpretability of the word-domains is methodologically very crucial in the study of the FSyst RWS-interpretability, as FSysts play a tool to simulate human capabilities in handling linguistic words in problem solving. This is also a basis that motivates the authors of [8] to propose the RWS-approach to the FSyst interpretability to show that the lack of a formal basis to connect fuzzy sets with the semantics of the words assigned to them is a crucial reason which makes the FSyst interpretability examined in the fuzzy set framework to become too sophisticated [8].

\subsubsection{The RWS-interpretability of HAs-Mathematical models of word-domains of variables}

Straight lines in reality and the human calculation demand based on their structures motivate the development of the theory of the real numbers. The successful applications of 
this theory in reality demonstrate that it is RWS-interpretable. So, methodologically, the RWS-interpretability of the theory of real numbers can be ensured by the following two facts:

- The RWS-interpretability of its axioms: It is well known that straight lines are RWmodels of the theory of the real numbers and, hence, its axioms that formulate the properties of the operations on the real numbers are compatible with the respective measurements made by human being on corresponding segments of a straight line. These demonstrate that the theory of the real numbers has a close connection with its RW-counterpart. Thus, the RWS-interpretability of its axioms are justified by RWsemantics representing distance relationships between real points on the straight lines.

- The development of the theory that is based on the inference rules of a formal logics does maintain the RWS-interpretability of the whole theory. The (logical) validity of a sentence is verified based on the RW-events. For instance, the validity of the sentence "If we have free time on Sunday AND there is sunshine on this day, then we will visit your family" is verified based on the respective events actually happen or do not happen in that day and, hence, it is RWS-interpretability. Since all inference rules preserve the validity of the sentences, it follows the validity of the mentioned above fact.

Based on these facts, we can show that the theory of HAs including their quantification are RWS-interpretable. Since the logical rules are the same as in the field of the mathematics, it is sufficient to show that the axioms of the HAs and their quantification are RWS-interpretable.

On one hand, human societies including their natural languages are evidently considered as a part of the RW. On other hand, as previously argued, natural languages are RWSinterpretable and, hence, the word-domains of the variables, which are ordered based on their inherent meaning and viewed as their sub-RW-parts, can also be considered as RWparts. Thus, to model the word-domains of variables, similarly as for the theory of the real numbers, the theory of HAs is developed in an axiomatic manner and their axioms are formal formulations of key essential properties of words and hedges of their respective word-domains $[5,6,7,9]$ viewed as RW-counterparts. As examined in [9], the quantification of HAs is also developed in axiomatic way and its axioms are established based on the structure of HAs. This ensures the RWS-interpretability of the quantification axioms.

In such a way, their RWS-interpretability is guaranteed by the hypothesis formulated above. So, similarly as for any classical math-theory, we have the following proposition.

Proposition 1. Any HA and its quantification theory are RWS-interpretable.

This claim is very important as we will see that the fuzzy sets theory is in general not RWS-interpretable, though this might not decrease the great meaning of this theory in application problem solving. This shows only that in the standpoint of the RWS-approach, the fuzzy set theory has some methodological shortcomings, emphasizing that its successful applications of the up to now demonstrate that the fuzzy sets theory is very elastic and flexible and based on this many powerful methods can be developed to solve application problems in various distinct disciplines. 


\section{RWS-INTERPRETABILITY OF THE FUZZY SYSTEMS COMPONENTS}

Since the RWS-interpretability in the sense of Def. 1 of the ordinary math-theories and the physical ones seems to be an evident natural requirement, their RWS-interpretability problem is not necessary to explicitly formulate. However, in the fuzzy linguistic environment it becomes a research topic in [8] which is motivated by the observation that there is a formalized gap between the RW-semantics of words and their computational semantics, including the fuzzy set based semantics, in the existing approaches. The semantics of the linguistic words, as symbolic expressions, of a variable is understood as a relationship between the linguistic words and the RW-objects that the words express. For the math- or physical theories, it is easy to specify these RW-objects, but for linguistic words it is not because the semantics of words are still not formally defined. For this, one has no formal foundation to examine whether linguistic words can represent certain RW-objects in reality. At the same time, the role of the natural languages in connecting with the RW has still not been taken into account.

This analysis shows that the RWS-approach is actually quite new and it is for the first time discussed in [8]. By this reason, in order to more easily understand the practical meaning of the scheme described in Fig. 2 and its application in Section 4, it is more useful to summarize here how one can solve the RWS-interpretability of the FSyst components examined in that paper. To properly follow Task 1-3 mentioned above, the main idea to ensure the RWS-interpretability of these components is to discover key structural features of the RW-semantics of the components that are described by human expert in terms of his own linguistic terminologies to formulate certain constraints to impose required restrictions on the constructed interpretation mappings to translate the components into their computational expressions in a computational space CS. So, differently from existing approaches, linguistic words actually play crucial and fundamental role in the RWS-approach.

A FSyst can be viewed as comprising a fuzzy knowledge base, which includes its linguistic frames of cognition (LFoC) and its linguistic fuzzy rule base (LRB), and a fuzzy inference engine, which is constructed mainly based on an approximate reasoning method (ARM).

\subsection{The RWS-interpretability of LFoCs}

In the fuzzy set framework, there is a concept of Frame of Cognition (FoC) which is any set of pre-specified fuzzy sets associated with an attribute/variable used as a set of descriptions to cognize the RW. Its fuzzy sets are always associated with words based only on human user intuition which are linearly ordered from left to right relying on their meaning. In the HA based approach (HA-approach) $[12,3,2]$ the set of these words is called a Linguistic Frame of Cognition ( $\mathrm{LFoC}$ ), denoted by $\mathfrak{F}$. In the fuzzy set framework, these fuzzy sets arranged in this way are considered as a fuzzy set representation of the set of words without any reasonable formal justification. In this situation, the interpretability of this fuzzy set representation of the LFoC $\mathfrak{F}$ is studied based only on expert intuition, i.e. the expert has to answer the question how he can arrange the shapes and the positions of the fuzzy sets on the variable universe in a meaningful way. Of course, these arrangements of fuzzy sets have no formal relation with the semantics of words.

In the RWS-approach, an RWS-interpretable computational representation of a given 
LFoC $\mathfrak{F}$ is constructed based on the scheme given in Fig. 2 described as follows, where $\mathfrak{F}=X_{(k)}=\{x \in X$ : the set of the words of a variable whose length is not greater than $k>0\}$.

\subsubsection{Try to discover structural relationships between words of $\mathfrak{F}$ being con- sidered as an $\mathbb{L} E$}

As previously discussed, $\mathfrak{F}$ can be regarded as a RW-counterpart (or, its words can properly convey their RW-semantics) and we try to find out key structural features of $\mathfrak{F}$. It can be seen that on this set there exist two relations, denoted by $\leq$ and $G S(x, y)$, which are still not considered in the fuzzy set framework.

$\circ \mathfrak{F}$ is linearly ordered set induced by the word meaning. Its structure is denoted as $(\mathfrak{F}, \leq)$.

- $G S(x, y)$ is a generality-specificity relation on $\mathfrak{F}$. For example, for the variable AGE, "old" is more general than "very_old", and "rather very young" is more specific than "young", if they all are in $\mathfrak{F}$. It can be verified that $G(x, y)$ have the following properties:

- Anti-symmetry: $G S(x, y) \& G S(y, x) \Rightarrow x=y$.

- Transitivity: $G S(x, y) \& G S(y, z) \Rightarrow G S(x, z)$.

These relations are taken as constraints imposed on two respective interpretation mappings of sound computational representations of LFoCs, where a sound computational representation of an LFoC means that these exists an explicit formal basis to believe that the computational objects of this representation can properly represent the semantics of the words appearing in the LFoC of a variable:

Definition 2. A fuzzy set representation of a given LFoC $\mathfrak{F}, F R(\mathfrak{F})=\{F(x): x \in \mathfrak{F}\}$, where $F(x)$ denotes the fuzzy set assigned to $x$, is said to be RWS-interpretable if the following conditions hold:

(i) On $F R(\mathfrak{F})$ can be defined two relations: the first one is denoted by $\leq^{*}$, which is reflexive, anti-symmetric and transitive, and the second one is denoted by $G S^{*}$, which is anti-symmetric and transitive.

(ii) There exists an interpretation assignment $I_{\leq}$and $I_{G S}$ that both map $\mathfrak{F}$ into $F R(\mathfrak{F})$, such that they respectively preserve the relations $\leq$ and $G S$ on $\mathfrak{F}$, i.e., for all $x, y$ in $\mathfrak{F}$, we have $x \leq y \Rightarrow I_{\leq}(x) \leq^{*} I_{\leq}(y)$ and $G S(x, y) \Rightarrow G S^{*}\left(I_{G S}(x), I_{G S}(y)\right)$.

This definition states that the RWS-interpretability of $F R(\mathfrak{F})$ can be ensured by imposing discovered constraints on the desired interpretation assignments. These constraints are so strong that there does not exist any fuzzy set representation of a given LFoC examined in recent literature that can be deemed as RWS-interpretable. That is, there do not exist interpretations induced by this representation that satisfy the conditions in Def. 2. So, to construct a RWS-interpretable computational representation of an LFoC $\mathfrak{F}$, one has to find a suitable topology (not only linear arrangement of the desired computational objects) to 
arrange the computational objects to represent $\mathfrak{F}$ so that the interpretation mappings $I_{\leq}$and $I_{G S}$ induced by the constructed computational representation of $\mathfrak{F}$ can meet the proposed constraints.

\subsubsection{Try to construct a computational space that can properly represent the semantics of $\mathfrak{F}$}

This is a difficult task as one has to find which computational space is appropriate, that is, one has to solve which math-objects can be used to represent the word semantics and, in the same time, to find suitable relations defined on the desired computational space to model the discovered relations $\leq$ and $G S$. Restricted to the triangle or trapezoid fuzzy sets, the study [8] argued that the topology of multi-granularity structure of fuzzy sets, as shown in Fig. 3 can meet both above discovered constraints, where the fuzzy sets are arranged in three level of their generality. Here, the fuzzy sets on the $k$ th-level represent the semantics of the words of the generality of degree $k$. For $k=0$, the words on this level are the most general and the fuzzy sets are either all triangles or all trapezoids, whore core are therefore represented by bolded points in the figure and whose supports are uniquely defined by the fuzziness intervals of the respective words. Representing triangles/trapezoids by triples of the form $(a, \boldsymbol{b}, d)$, where $\boldsymbol{b}$ 's are the cores of triangles/trapezoids, the order between the fuzzy sets are defined as follows: $(a, \boldsymbol{b}, d) \leq^{*}\left(a^{\prime}, \boldsymbol{b}^{\prime}, d^{\prime}\right) \Leftrightarrow\left\{\boldsymbol{b} \leq \boldsymbol{b}^{\prime} \&\right.$ there is at least one of the remaining components of the triples, say the first one, satisfying the inequality $\left.a \leq a^{\prime}\right\}$. The relation $G S^{*}$ between the triples are defined as follows: $G S^{*}\left((a, b, d),\left(a^{\prime}, \boldsymbol{b}^{\prime}, d^{\prime}\right)\right) \Leftrightarrow$ $[a, d] \subseteq\left[a^{\prime}, d^{\prime}\right]$. Obviously, the triples on the top level are of the most generality which is compatible with the semantics of the words on this level.

Note that, in the existing approaches in the fuzzy set framework, the fuzzy sets are usually arranged to form a single-granularity structure, i.e. they are arranged on a unique level instead of the three levels as in the case of Fig. 3. That is, all their associated words are on the same level of generality (vs. specificity) and, hence, in this topology, they are in general not able to represent the relation $G S$.

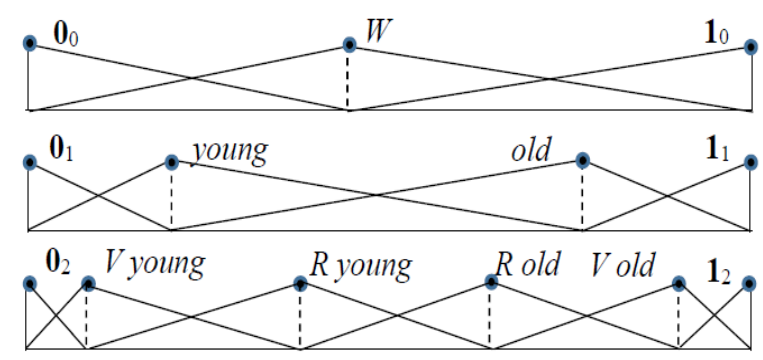

Figure 3. RWS-Interpretable triangle/trapezoid multi-granular representation of $X_{A G E,(2)}$

\subsection{The RWS-interpretability of computational representation of LRBs and ARMs}

The concept of the RWS-interpretability of FSysts or, more generally, of formalized expressions is very complex and of high abstract. In this section, the RWS-interpretability 
of LRBs and ARMs will be analyzed with more exact and confident explanation and a modification of the concept of the RWS-interpretability of computational LRB-representation and ARMs examined in [8] is given here. E.g. as an ARM must be developed based on a given method producing a computational representation of any given LRB, the RWSinterpretability of the computational representation of LRBs and of an ARM to be developed to work on it will be defined in a close relation to each other.

In [8], it is argued that one may acquire a piece of knowledge about a numeric dependent relationship between two variables only when it is observed that they are monotonically dependent on each other on a certain interval of each variable, otherwise their dependence is chaotic. Compatibly with this, as discussed in that study, it is interesting that the semantics of a multi-variable linguistic rule does also express monotonic dependent relationships between its unique output variable and one of its input variables. For example, in case of one output and one input variable, it is easily observed that the rule "IF $\operatorname{AGE}(a)$ is young, THEN RUN_SPEED $(a)$ is fast" represents an inversely proportional relationship between AGE and RUN_SPEED of a person $a$, while "IF HIGH $(a)$ is tall, THEN RUN_SPEED $(a)$ is fast" represents a directly proportional one.

Given the above two sentence with their own RW-semantics, we may intuitively deduce the following linguistic rule with two input variables, where 'More' is viewed as a hedge [5, 6]:

"IF $\operatorname{AGE}(a)$ is young $\operatorname{AND} \operatorname{HIGH}(a)$ is tall, THEN RUN_SPEED $(a)$ is More _ fast"

This sentence can be considered as an aggregation of the two given sentences above. More generally, the following linguistic rule of one output and $m$ input variables,

$(r) \quad$ IF $\mathcal{X}_{1 L}$ is $x_{1} \& \ldots \& \mathcal{X}_{m L}$ is $x_{m}$ THEN $\mathcal{X}_{m+1, L}$ is $x_{m+1}$

where, by our convention, $\mathcal{X}_{j L}$ 's are its linguistic variables of the respective given real-world variables $\mathcal{X}_{j}$ 's, $j=1$ to $m+1$. The rule $r$ can be considered as an aggregation of rules 'IF $\mathcal{X}_{j L}$ is $x_{j}$ THEN $\mathcal{X}_{m+1, L}$ is $x_{m+1}, j=1$ to $m+1$, and, thus, $r$ represents $m$ monotonic relationships of $\mathcal{X}_{m+1, L}$ and $\mathcal{X}_{j L}$ on certain interval of each variable, for $j=1$ to $m$. This semantics of linguistic rules is very crucial but the fuzzy rules in the fuzzy set framework do not own such a semantics. It is even more crucial that the above monotonic relationships defined by $r$ given in (1) represent similar relationships between the corresponding real-world variables $\mathcal{X}_{j}$ 's and, hence, they are compatible with the respective relationships between the corresponding numeric variables $\mathcal{X}_{j N}$ 's, $j=1$ to $m+1$.

To further analyze, we assume that a LRB $\mathcal{R B}$ is full of conditions, that is, all $m$ variables $\mathcal{X}_{j L}, j=1, \ldots, m$, are explicitly present in each rule (as it happens with the well-known Wang and Mendel rules ([?]). In this case, it is simply called a full LRB. It is natural to require $\mathcal{R B}$ to be consistent, i.e. if the antecedents of any two rules of $\mathcal{R B}$ are identical, then so are their conclusions. Then, a full and consistent LRB $\mathcal{R B}$ may be considered as representing a linguistic functional dependence of $\mathcal{X}_{m+1, L}$ on $\mathcal{X}_{j L}$ 's, $j=1$ to $m$. By the RWSinterpretability of natural languages and the RW-semantics of linguistic rules and LRBs, $\mathcal{R B}$ represents a real-world functional dependence of $\mathcal{X}_{m+1}$ on $\mathcal{X}_{j}$ 's, for $j=1$ to $m$.

Such a RW-semantics of LRBs is very important for the examination of the RWSinterpretability of computational representations of LRBs and of ARMs which work on RWS-interpretable computational representation of the given LRBs. Note that in numerous applications, an ARM working on an LRB with $m$ input variables is required to produce a numeric output for a given numeric input vector $\left(a_{1}, \ldots, a_{m}\right)$. Since the ultimate purpose of a FSyst equipped with a LRB is to interact with its real-world counterpart to solve an 
application problem, the RWS-interpretability of the LRB is to guarantee that the produced real numeric output value by the system, for a given real numeric input vector, is just the one the designer expects. This requirement is essential and, therefore, following the diagram given in Fig. 2, one has to determine a proper part of the real-world which a given LRB does represent to discover its natural and essential real-world properties from which constraints imposed on the LRB are discovered. As analyzed just above, the RW-semantics of a given LRB, $\mathbb{B}$, expresses a RW-functional dependence of $\mathcal{X}_{m+1}$ on $\mathcal{X}_{j}$ 's, $j=1$ to $m$, which is monotonic with respect to every individual input variable on a certain interval of its real universe. In reality, the RW-semantics of $\mathbb{B}$ is also modeled by a numeric functional dependence of $\mathcal{X}_{m+1, N}$ on $\mathcal{X}_{j N}$ 's, for some $j$, which must be monotonic on the observed intervals of its two variables. This real-world semantics induces necessary constraints related to the monotonicity of $\mathbb{B}$ imposed on a method assigning $\mathbb{B}$ to a computational object $\mathcal{C}(\mathbb{B})$ and on a ARM $\mathrm{R}$ working on $\mathcal{C}(\mathbb{B})$ that can be formulated in a close relation as follows.

In the fuzzy set framework, $\mathbb{B}$ can be represented by a fuzzy relation $\mathcal{R}$ defined in Cartesian product $U_{1} \times \ldots \times U_{m+1}$ that is produced a certain representation method. More generally, we deal with LRBs instead of FRBs. In general, there are several computrepresentation methods (CRep-methods) of LRBs. Every such a CRep-method, $\mathcal{M}$, should transform any LRB $\mathbb{B}$ of rules of the form (1) into a desired comput-object that represents the computational semantics of the given LRB in a way as follows:

(i) Construct an interpretation $I_{\mathcal{X}}$ that maps words of $\mathcal{X}_{j}$ to a desired comput-space $C S_{j}$, $j=1$ to $m$;

(i) Construct a procedure $P$ that maps connectives present in $\mathbb{B}$ to their operations of a space $\mathcal{S}=\left(\mathcal{C}, \leq_{\mathcal{S}}\right)$ defined on Cartesian product $C S_{1} \times \ldots \times C S_{m+1}$, where $\left(C S_{j}\right.$, $\leq_{j}$ )'s are order-based structures, so that the composition $P \circ\left(I_{\mathcal{X} 1}, \ldots, I_{\mathcal{X} m+1}\right)$ transforms $\mathbb{B}$ into an object of $\mathcal{S}$ and $\mathbb{B}$ may be a unique rule $r$.

For illustration, the fuzzy relation $\mathcal{R}$ defined by Mamdani's method to model a LRB $\mathbb{B}$ of $n$ rules can be rewritten as

$$
\left(P \circ\left(I_{x_{1}}, \ldots, I_{x_{m+1}}\right)\right)(\mathbb{B})=\bigcup_{1 \leq i \leq n} I_{x_{1}}\left(x_{i, 1} \cap \ldots \cap I_{x_{m+1}}\left(x_{i, m+1}\right)=\bigcup_{1 \leq i \leq n}\left(P \circ\left(I_{x_{1}}, \ldots, I_{x_{m+1}}\right)\right)\left(r_{i}\right)\right.
$$

where $I_{\mathcal{X} j}$ 's assign the words of their variables to their fuzzy sets of the respective variable universes.

$\mathcal{M}$ defines a mapping which maps any LRB $\mathbb{B}$ to a comput-representation $\mathcal{M}(\mathbb{B})$ of $\mathbb{B}$ in $\mathcal{S}$. By $\left.\mathcal{M}(\mathbb{B})\right|_{\mathcal{X}_{j}}$ we denote the projection of $\mathcal{M}(\mathbb{B})$ on the universe of $\mathcal{X}_{j}$. The following definition is more formally defined than that given in [8].

Definition 3. Given comput-space $\mathcal{S}=\left(\mathcal{C}, \leq_{\mathcal{S}}\right)$ defined on Cartesian product of order-based structures $C S_{j}^{\prime}$ s. A CRep-method $\mathcal{M}$ with its interpretations $I_{\mathcal{X}}^{\prime} \mathrm{s}, I_{\mathcal{X}_{j}}: \operatorname{Dom}\left(\mathcal{X}_{j}\right) \rightarrow C S_{j}$, is said to be RWS-interpretable in $\mathcal{S}$ provided that

1) The interpretations $I_{\mathcal{X}}^{\prime}$ s are order isomorphisms.

2) For a given $\operatorname{LRB} \mathbb{B}, \mathcal{M}$ preserves the discovered monotonicity, if any, of $\mathbb{B}$. That is, if $\mathbb{B}$ is increasing (or decreasing) and for $\boldsymbol{a}=\left(x_{i 1}, \ldots, x_{i m}\right) \leq \boldsymbol{a}^{\prime}=\left(x_{i^{\prime} 1}, \ldots, x_{i^{\prime} m}\right)$, where 
$\boldsymbol{a}$ and $\boldsymbol{a}^{\prime}$ are any two linguistic vectors formed by $m$ words appearing in, respectively, some two rules $r_{a}$ and $r_{a^{\prime}}$ of $\mathbb{B}$, then $\left.\mathcal{M}\left(r_{a}\right)\right|_{\mathcal{X}_{m+1}} \leq\left.\mathcal{M}\left(r_{a^{\prime}}\right)\right|_{\mathcal{X}_{m+1}}\left(\right.$ or, $\left.\mathcal{M}\left(r_{a}\right)\right|_{\mathcal{X}_{m+1}} \geq$ $\left.\left.\mathcal{M}\left(r_{a^{\prime}}\right)\right|_{\mathcal{X} m+1}\right)$.

It can be observed in general that any method that represents any LRB as an $(m+1)$ dimensional fuzzy relation cannot be RWS-interpretable, as the order of words and their fuzzy sets is ignored.

The ARMs developed to solve application problems play a key role to construct effective but also interpretable FSysts. They strongly depend on a given LRB $\mathbb{B}$ itself as well as also on the proposed method $\mathcal{M}$ which produces a computational representation of $\mathbb{B}$. As the developed ARM R must be developed to work on the computational representation of $\mathbb{B}$ produced by an $\mathcal{M}$, its RWS-interpretability is strongly dependent on $\mathcal{M}$. Thus, the RWS-interpretability of an AMR should be defined based on a given the RWS-interpretable CRep-method $\mathcal{M}$. Now, we are ready to introduce the following definition, where for a given vector $\boldsymbol{a}=\left(a_{1}, \ldots, a_{m}\right), \mathbb{R}(\boldsymbol{a})$ denotes the numeric output value of $\boldsymbol{a}$ produced by $\mathbb{R}$.

Definition 4. Assume that an ARM $\mathbb{R}$ is developed to work on the LRB-representations produced by a given CRep-method $\mathcal{M}$. Then, $\mathbb{R}$ is said to be $R W S$-interpretable provided that for any LRB $\mathbb{B}$ which is monotonic with respect to every individual input variable of $\mathbb{B}$, it satisfies the following condition

$$
\left.\left(\forall \boldsymbol{a}, \boldsymbol{a}^{\prime}\right)\left\{\left[\boldsymbol{a} \quad \boldsymbol{a}^{\prime} \Rightarrow \mathbb{R}_{\mathcal{M}(\mathbb{B})}(\boldsymbol{a}) \leq \mathbb{R}_{\mathcal{M}(\mathbb{B})}\left(\boldsymbol{a}^{\prime}\right)\right] \text { and }\left[\boldsymbol{a} \neq \boldsymbol{a}^{\prime}\right) \Rightarrow \mathbb{R}_{\mathcal{M}(\mathbb{B})}(\boldsymbol{a}) \neq \mathbb{R}_{\mathcal{M}(\mathbb{B})}\left(\boldsymbol{a}^{\prime}\right)\right]\right\}
$$

\section{THE RWS-INTERPRETABILITY CONCEPT OF LINGUISTIC FUZZY EXPRESSIONS/THEORIES}

As discussed in Section 2 and 3, the novel RWS-interpretability of any fuzzy procedures/theories, in general, and of any FSysts, in particular, seems to be very essential and practical. So, a natural question that arises is that whether the fuzzy sets theory or its expressions are RWS-interpretable and if it is not, whether there exist methodologies to develop RWS-interpretable FSysts?

\subsection{Examination of the RWS-interpretability of some fuzzy expressions of the fuzzy set theory}

The RWS-interpretability of the fuzzy set theory is too big a problem and, therefore, in this section it is restricted to examine the RWS-interpretability of the standard fuzzy set algebras.

\subsubsection{An analysis of the RWS-interpretability of the standard fuzzy set algebra}

Let us consider a universe $U$ and denote by $F(U)$ the set of all fuzzy sets of $U, F(U)=$ $\left\{\mu: \mu \in[0,1]^{U}\right\}$, where $[0,1]^{U}$ is the set of all (membership) functions from $U$ into $[0,1]$ and the fuzzy sets and their membership functions can be viewed as being identical. It is wellknown that the union $(\cup)$, intersection $(\cap)$, complementation $(\sim)$ can be defined in $F(U)$ as a generalization of the respective operations on the crisp sets of $U$. They are pointwise defined on the membership functions of the fuzzy sets in the whole $F(U)$. Then, we have a standard fuzzy set algebra that can be denoted by $F \mathbb{A}=(F(U), \cup, \cap, \sim)$. 
To examine the RWS-interpretability of $F \mathbb{A}$, we have to find out which is the RWsemantics of individual fuzzy sets and the RW-semantics of the operations $\cup, \cap$ and $\sim$ when they act on fuzzy sets. To answer these questions, we should come back to the ultimate aim of the development of the fuzzy sets theory: to simulate human capabilities in handing words. This is why in applications the operations $\cup, \cap$ and $\sim$ are usually interpreted as representing the computational semantics of the respective logical connectives in natural languages, AND, OR and NOT. So, we will examine the RWS-interpretability of the operations of the standard fuzzy set algebra based on the real-world semantics of the connectives AND, OR and NOT.

There are two main reasons that show that $F \mathbb{A}$ can not be RWS-interpretable.

1) A methodological reason present in the fuzzy set framework. Let us consider the variable HIGH of people of a community and the meaning of the sentence "he is "Tall OR Rather_tall"'. The semantics of this sentence must be considered in the context of the wordexpressions of the word-domain of HIGH, $L D o m(H I G H)$. Assume that the standard fuzzy set algebra defined on the universe $U$ of HIGH is $F \mathbb{A}=(F(U), \cup, \cap, \sim)$. In principle, to simulate human capabilities in handling linguistic words, the fuzzy set based semantics of the words of $\operatorname{LDom}(\mathrm{HIGH})$ is defined by an interpretation mapping $\Im: L D o m(\mathrm{HIGH}) \rightarrow F \mathbb{A}$ in such a way that $\Im$ can convey the RW-semantics of the own worlds of HIGH and of the linguistic expressions, say 'Tall OR Rather_tall', to the structure of the fuzzy set algebra FA, with its own formalism, which closely depends on the nature of the computational objects (fuzzy sets, here) and the way one can manipulate the fuzzy sets in the fuzzy set algebra $F \mathbb{A}$, i.e. it closely depends on the computational structure of $F \mathbb{A}$. Mathematically, it can easily be seen that

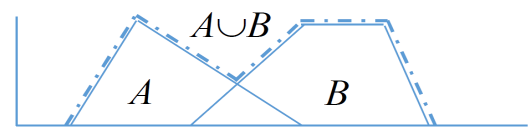

Figure 4. The union of the two given fuzzy sets of variable HIGH

Proposition 2. As $L D o m(\mathrm{HIGH})$ is finite, while $F(U)$ is innumerable and even of continuum power, there does not exist an interpretation mapping $\Im$ from $L D o m(\mathrm{HIGH})$ into $F(U)$ that can maintain the relationships that characterize the structure of $L D o m(\mathrm{HIGH})$, noting that the operations of $F \mathbb{A}$ are defined in the whole $F(U)$.

Proof. If $\Im$ can preserve the relationships of $\operatorname{LDom}(\mathrm{HIGH})$, the set $\Im(\operatorname{LDom}(\mathrm{HIGH})) \subseteq F(U)$ must be closed with respect to the connectives (i.e. operations) defined on $L D o m(\mathrm{HIGH})$. However, the fuzzy set operations cannot be defined in the finite set $\Im(\operatorname{LDom}(\mathrm{HIGH}))$ of fuzzy sets associated with the words in $L D o m(\mathrm{HIGH})$. For example, the union of the two fuzzy sets $A$ and $B$ defined in the whole $F(U)$ is represented by the dashed line in the Fig. 4, which is formed by just segments of the border lines of $A$ and $B$. The semantics of the wordsexpression " $w_{A}$ AND $w_{B}$ ", where the words $w_{A}$ and $w_{B}$ are assigned respectively to $A$ and $B$, means that it is an expression which dominates the both $w_{A}$ and $w_{B}$. So, the interpretation mapping preserves these relationships only when $A \cup B$ belongs to $\Im(L D o m(\mathrm{HIGH}))$, which in general is impossible.

2) A methodological reason on standpoint of the RWS-approach. First, we adopt an assumption that we deal with only variables with numeric linear universe and hence the worddomains of their linguistic variables are linearly ordered. So, their respective HAs are also 
linear.

In Section 2, it is shown by Proposition 1 that the HA $A X_{H I G H}$ is RWS-interpretable. That is there exists an interpretation mapping $\Im_{H I G H}$ from LDom(HIGH) into the underlying set of $A X_{H I G H}$, which implies that $\Im_{H I G H}\left(w_{A}\right.$ AND $\left.w_{B}\right)=\Im_{H I G H}\left(w_{A}\right) \vee \Im_{H I G H}\left(w_{B}\right)$, where $\vee$ is joint operation defined in the order-based structure $A X_{H I G H}$. Since $A X_{H I G H}$ is RWS-interpretable, $\Im$ preserves the relationships between words of $L D o m(\mathrm{HIGH})$, the expression $\Im_{H I G H}\left(w_{A}\right) \vee \Im_{H I G H}\left(w_{B}\right)$ represents the semantics of the word-expression " $w_{A}$ AND $w_{B} "$. As $A X_{H I G H}$ is linear, $\Im_{H I G H}\left(w_{A}\right) \vee \Im_{H I G H}\left(w_{B}\right)=\max \left\{\Im_{H I G H}\left(w_{A}\right), \Im_{H I G H}\left(w_{B}\right)\right\}$ and it represents the RW-semantics of the expression " $w_{A}$ AND $w_{B}$ ". Since as mentioned above, the fuzzy sets $A$ and Bassociated with respectively the word $w_{A}$ and $w_{B}$, but $A \cup B \notin\{A, B\}$, which is not compatible with $\Im_{H I G H}\left(w_{A}\right) \vee \Im_{H I G H}\left(w_{B}\right)=\max \left\{\Im_{H I G H}\left(w_{A}\right)\right.$, $\left.\Im_{H I G H}\left(w_{B}\right)\right\}$ which represents the RW-semantics of " $w_{A}$ AND $w_{B}$ ". This asserts that the standard fuzzy set algebra $F \mathbb{A}$ is not RWS-interpretable.

\subsubsection{A discussion of the RWS-interpretability of Mamdani fuzzy reasoning method}

In Mamdani fuzzy reasoning method which is denoted by $\mathrm{ARM}_{M m d}$, its fuzzy rule base (FRB), $\mathbb{B}$ consists of $n$ rules of a similar form as in (1), but at the positions of the words $x_{j k}$ 's are fuzzy set $f\left(x_{j k}\right)$ 's assigned to the words denoted also by $x_{j k}$ 's

$(\mathbb{B})$ IF $\mathcal{X}_{1 L}$ is $f\left(x_{1, k}\right) \& \ldots \& \mathcal{X}_{m L}$ is $x_{m i, k}$, THEN $\mathcal{X}_{(m+1) L}$ is $x_{(m+1), k}$, for $k=1$ to $n$ (4)

Note that, in the fuzzy set framework, linguistic words are only linguistic labels intuitively assigned by human user to the fuzzy sets under consideration. They do not play any role in formally handling their associated fuzzy sets. So, there is no formal basis to connect the designed fuzzy sets with their assigned words.

Table 1. Simplified FRB for the actuator on the $1^{\text {th }}$-storey

\begin{tabular}{|l|l|l|l|}
\hline \multicolumn{1}{|c|}{$x_{2} \dot{x}_{2}$} & NS & $Z$ & PS \\
\hline NS & NM & NS & Z \\
\hline$Z$ & NS & Z & PS \\
\hline PS & Z & PS & PM \\
\hline
\end{tabular}

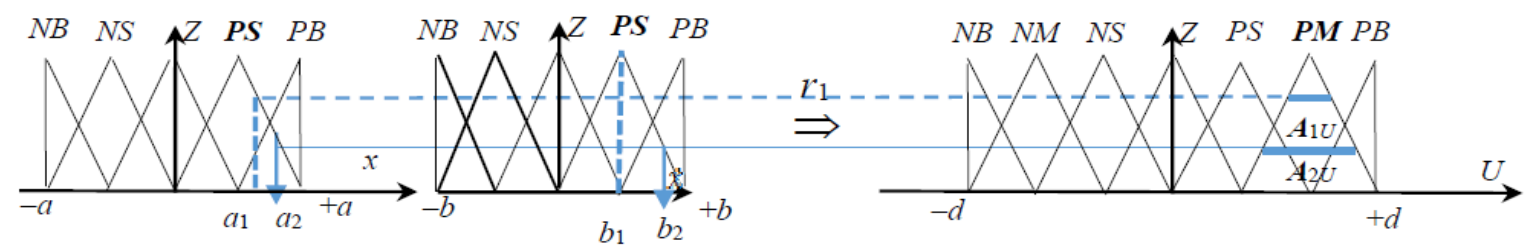

Figure 5. Computational representation of the rules $r_{1}$ and $r_{15}$ of the LRB $\mathfrak{B}$ given in Tab. 1

Let us consider an FRB, $\mathfrak{B}$, given in Tab. 1, consisting of 9 fuzzy rules, that is simplified from the one having 25 rules given in [3]. The fuzzy sets associated with linguistic labels are represented in Fig. 5 which are plotted similarly as in [3] with a note that in practice the linguistic labels under consideration are not required to be present in the FRB $\mathfrak{B}$. The constructed fuzzy sets associated with the linguistic labels absent in $\mathfrak{B}$ contribute to define 
the desired fuzzy-set-based semantics of all the linguistic words under consideration. It is observed that it describes a linguistic function whose linguistic labels of the linguistic variable $U_{L}$ increasingly depend on the linguistic labels of every input linguistic variable $X_{1 L}$ or $\dot{X}_{1 L}$. Since, by our hypothesis, every human language is RWS-interpretable, the given FRB $\mathfrak{B}$ describes a RW-function $u=f\left(x_{1}, \dot{x}_{1}\right)$ which is also increasingly dependent on every variable $x_{1}$ or $\dot{x}_{1}$, when regarding $U, X_{1}$ and $\dot{X}_{1}$ as RW-variables. This discovered increasing dependence must be considered as a constraint on any applied to the given $\mathfrak{B}$.

The $\mathrm{ARM}_{M m d}$ can be described as follows:

- Construct fuzzy sets associated with the linguistic labels of every linguistic variable appearing in the given FRB. Assume that they are constructed as shown in Fig. 5, which represents a fuzzy rule $r_{1}$ formulated by "IF $X$ is PS \& $\dot{X}$ is PS THEN $U$ is PM", where the linguistic labels of $r_{1}$ are bolded in the figure. The 8 remaining fuzzy rules are represented in a similar way.

- For every numeric input vector, say $\left(a_{1}, b_{1}\right)$, it fires the antecedent components of a given rule under consideration, say $r_{1}$ in Fig. 5, and this rule produces a numeric output calculated in the following way: Denote by $\mu_{X, P S}$ and $\mu_{\dot{X}, P S}$ the membership functions of the fuzzy sets associated, respectively, with PS of $X$ and with PS of $\dot{X}$. The values $a_{1}$ and $b_{1}$ of the input vector $\left(a_{1}, b_{1}\right)$ fire respectively the fuzzy set with $\mu_{X, P S}$ and the one with $\mu_{\dot{X}, P S}$. The firing degrees are respectively $\mu_{\dot{X}, P S}\left(a_{1}\right)$ and $\mu_{\dot{X}, P S}\left(b_{1}\right)$. The smaller firing degree among them is $\mu_{X, P S}\left(a_{1}\right)$ which is conveyed to cut the fuzzy set $\mu_{U, P M}$ of $U$ on the right side of $r_{1}$ at the level $\mu_{X, P S}\left(a_{1}\right)$ and, in this way, the rule $r_{1}$ produces an output fuzzy set $\boldsymbol{A}_{1 U}\left(a_{1}, b_{1}\right)$.

Similarly, the input vector fires $\left(a_{1}, b_{1}\right)$ also the rules $r_{j}$ of the given by Tab. 1 , for $j$ $=1$ to 8 , and $r_{j}$ produces a fuzzy set output, denoted by $\boldsymbol{A}_{j U}\left(a_{1}, b_{1}\right)$, for $j=1$ to 8 . It should make a note that $a_{1}$ fires also the fuzzy set of $\mathrm{PB}$, but there is no rule in $\mathfrak{B}$ that has PB in its antecedent.

- The output fuzzy set $\boldsymbol{A}_{U},\left(a_{1}, b_{1}\right)$ produced from the FRB $\mathfrak{B}$ and from the input vector $\left(a_{1}, b_{1}\right)$ is defined by $\boldsymbol{A}_{U, \mathfrak{B}}\left(a_{1}, b_{1}\right)=\bigcup_{1<j<15} \boldsymbol{A}_{j U}\left(a_{1}, b_{1}\right)$. Its corresponding numeric output of $\left(a_{1}, b_{1}\right)$, denoted by $u_{1},\left(a_{1}, b_{1}\right)$, is determined by the centroid defuzzification method applied to $\boldsymbol{A}_{U, \mathfrak{B}}\left(a_{1}, b_{1}\right)$.

To answer the question that whether $\mathrm{ARM}_{M m d}$ is RWS-interpretable, we prove the following.

Theorem 1. Mamdani's method ARM Mmd is not RWS-interpretable.

Proof. Since any ARM has to be applied to any FRBs, to prove that $\mathrm{ARM}_{M m d}$ is not RWSinterpretable, it is required that it does not satisfy constraint (3) in Def. 3 when it is run on a monotonic FRB, say the given $\mathfrak{B}$ in Tab. 1. To verify this, let us consider two input vectors $\boldsymbol{v}_{1}=\left(a_{1}, b_{1}\right)$ and $\boldsymbol{v}_{2}=\left(a_{2}, b_{2}\right)$, whose component values are located as shown in Fig. 5. It is observed that they satisfy the inequality $\boldsymbol{v}_{1 i} \boldsymbol{v}_{2}$ and, since the values $a_{1}$ and $a_{2}$ cannot fire the fuzzy sets of $\mathrm{N}$ and $\mathrm{Z}$ (their firing degrees are zero) and the same for the values $b_{1}$ and $b_{2}$.

So, only the $9^{t h}$-rule, $\mathrm{PS}_{X} \& \mathrm{PS}_{\dot{X}} \Rightarrow \mathbf{P M}$, is fired and its output fuzzy sets with the inputs $\boldsymbol{v}_{1}$ and $\boldsymbol{v}_{2}$ are, respectively, $\boldsymbol{A}_{1 U}$ and $\boldsymbol{A}_{2 U}$, as plotted in Fig. 5. Note that the high of 
the trapezoid $\boldsymbol{A}_{1 U}$, denoted by $h\left(\boldsymbol{A}_{1 U}\right)$, is greater than the high $h\left(\boldsymbol{A}_{1 U}\right), h\left(\boldsymbol{A}_{1 U}\right)>h\left(\boldsymbol{A}_{1 U}\right)$. These imply that the out numeric values of $\boldsymbol{v}_{1}$ and $\boldsymbol{v}_{2}$ are equal and the condition (3) is not satisfied. Therefore, Mamdani's method $\mathrm{ARM}_{M m d}$ is not RWS-interpretable, by Def. 3 .

\subsection{Discuss the RWS-interpretability of graphic representation of LRBs and of the interpolation reasoning HA-based method}

A question arising is whether there exists an RWS-interpretable ARM? In principle, this problem depends also on the RWS-interpretability of the theories/methodologies based on which ARMs are constructed. Since it is a complex problem, in this study we are restricted in the concrete question saying that are there a computational representation of the FRB $\mathfrak{B}$ and an ARM which are RWS-interpretable? In this section, we will follow the HA approach to the inherent order-based semantics of words and the inherent semantic structures of worddomains of variables. The computational semantics of the words of a word-domain of a variable is developed based on a principle that the inherent order-based semantics of words does determine their computational semantics. Since this approach establishes a formalism to immediately handle the variable words, we use the terminology linguistic rules (or, linguistic rule bases (LRBs)) instead of fuzzy rules (or, fuzzy rule bases (FRBs) to emphasize this linguistic characteristic.

There are three basic quantitative semantics of the words of every variable $X$, which are defined closely to each other: the fuzziness measure, the fuzziness intervals (as being the interval-semantics) and the semantically quantifying mapping (SQM) of the variable word-domain which is uniquely determined by giving the numeric values of the independent fuzziness parameters of the variable. The SQM-values of the words are called the numeric word semantics. However, in this section, we utilize only SQMs which are characterized by the properties that they are order isomorphisms, i.e. they preserve the order relationships between the words, and their images of their corresponding variable domains are dense in the respective variable reference domains.

\subsubsection{RWS-interpretable computational representation of $\mathfrak{B}$}

On the mathematical point of view, when word-domains are formalized to become mathstructures, every linguistic rule of the form given in (4) can be considered as a point in the respective linguistic $(m+1)$-dimensional Cartesian space. So, every given LRB of the form (4) can be considered as modeling a linguistic function of $m$ variables going through these $n$ points, called graphic representation of (4). Then, an interpolation ARM to work with the given LRB can be developed as follows:

- For every variable $X_{j}, j=1, \ldots, m+1$, construct a $H A A X$ whose primary/atom words and hedges are compatible with the given LRB.

- Provide the fuzziness parameter values for each $X_{j}$ based on human user experience and establish the SQM $f_{j}$ which maps the words appearing in the LRB into the normalized reference domain $[0,1]$. Then, $n$ linguistic points mapped into $\mathrm{n}$ points in the Euclid space $[0,1]^{m+1}$, which are regarded as determining a numeric graph going through these points that simulates the above linguistic function. 
- Since the established SQMs are order isomorphisms, such a numeric graph can be considered as being similar to the linguistic graph defined by the given LRB $\mathfrak{B}$ in terms of the semantic order relations of each individual linguistic variable.

It is worth emphasizing that this function interpreted as a computational representation of $\mathfrak{B}$ is compatible with the RW-function between the respective RW-variables described by the given LRB $\mathfrak{B}$ in terms of monotonic relationships between variables. At the same time, it is also compatible with the numeric function which models the same above RW-function in terms of the relationships between the respective numeric variables of the aforementioned RW-variables. This is a specific, important and useful feature of the proposed graphic representation of LRBs

This is a formal basis to suggest us to apply a numeric interpolation method on this graphic representation of LRBs which is a numeric function graph in the space $[0,1]^{m+1}$.

\subsubsection{A linear interpolative ARM developed to work on the graphical repre- sentation of LRB}

Applying this interpolation ARM to the LRB $\mathfrak{B}$ given in Tab. 1 , since $\mathfrak{B}$ describes an increasingly monotonic linguistic function of two dimensions, its graph can be represented as in Fig. 6 which also simulates an increasingly monotonic numeric function going through 9 points in $[0,1]^{3}$ whose minimal value is located at $(0.18,0.18)$ and the maximal one is located at $(0.73,0.73)$. An ARM proposed here is developed based on ordinary linear interpolation on this graphical representation by establishing linear equations representing triangular sections [1] plotted in Fig. 6. Obviously, these linear equations represents linear dependences of $U$ on the input variables $x$ and $\dot{x}$ and, hence, the ordinary linear interpolation method ensures that the developed ARM satisfies the condition (3) of Def. 3. That is, it is RWS-interpretable.

\section{CONCLUSIONS}

The RWS-approach to the interpretability of FSysts for the first time proposed in [8] is quite novel and it is necessary to discussed with more details. It is argued in that paper that while the RW-semantics of the theories in mathematics and physics is very essential, though it is not explicitly declared, for applying these theories in applications and they are WRS-interpretable, in the fuzzy set framework the interpretability of methods or theories in this sense is still not under consideration. Methodologically, the RWS-interpretability of a formal theory is a distinguished characteristic to ensure that the behavior of a method developed based on this theory to solve a RW-problem can interact with its RW-counterpart properly as human expectation. This shows the important role of this concept and, thus, it is an indispensable characteristic of FSysts.

Motivated by this discussion and analysis, in this study, we aim first to more generally and clearly discuss and analyze that the RWS-interpretability is a necessary and general concept not only for FSysts, but also for any symbolic languages, theories, methods, ..., including ordinary basic theories of math or physics and event for social-economic theories. The RWS-interpretabilities of the two basic concepts, the LRB-Rep methods and ARMs are more exactly formalized. 
Restricted to the fuzzy linguistic environment, we show that

(i) It is initially proved that the fuzzy set theory involves fundamental parts which are not RWS-interpretable, e.g. the standard fuzzy sets algebra or fuzzy reasoning methods, while the theory of hedge algebras and their quantification theory are RWSinterpretable, similarly as math-theories.

(i) Meanwhile, the graphic representation of LRBs and ARM developed based on linear interpolation are proved to be RWS-interpretable.

\section{ACKNOWLEDGEMENTS}

This research is funded by Vietnam Academy of Science and Technology (VAST) under Grant No. VAST01.07/17-18 and by Viet Nam National Foundation for Science and Technology Development (NAFOSTED) under Grant No. 102.01-2017.06.

The authors gratefully acknowledge reviewers' comments improving better the paper.

\section{REFERENCES}

[1] H.-L. Bui, T.-A. Le, and V.-B. Bui, "Explicit formula of hedge-algebras-based fuzzy controller and applications in structural vibration control," Applied Soft Computing, vol. 60, pp. 150-166, 2007.

[2] Y. Dong, Y. Xu, and S. Yu, "Computing the numerical scale of the linguistic term set for 2-tuple fuzzy linguistic representation model," IEEE Trans. Fuzzy Syst., vol. 17, no. 6, pp. 1366-1378, 2009.

[3] R. Guclu and H. Yazici, "Vibration control of a structure with atmd against earthquake using fuzzy logic controllers," Journal of Sound and Vibration, vol. 318, no. 1, pp. 36-49, 2008.

[4] F. Herrera and L. Martínez, "A 2-tuple fuzzy linguistic representation model for computing with words," IEEE Trans. Fuzzy Syst., vol. 8, no. 6, pp. 746-752, 2000.

[5] N. C. Ho and W. Wechler, "Hedge algebras: an algebraic approach to structures of sets of linguistic domains of linguistic truth variables," Fuzzy Sets and Systems, vol. 35, no. 3, pp. 281-293, 1990.

[6] - "Extended hedge algebras and their application to fuzzy logic," Fuzzy Sets and Systems, vol. 52, no. 3, pp. 259-281, 1992.

[7] N. C. Ho and H. V. Nam, "An algebraic approach to linguistic hedges in zadeh fuzzy logic," Fuzzy Set and System, vol. 129, no. 1, pp. 229-254, 2002.

[8] N.C.Ho and J. Alonso, "Looking for a real-world-semantics-based approach to the interpretability of fuzzy systems," FUZZ-IEEE 2017 Technical Program Committee and Technical Chairs, July 9-12, 2017.

[9] N.C.Ho and V.L.Nguyen, "Fuzziness measure on complete has and quantifying semantics of terms in linear hedge algebras," Fuzzy Set and System, vol. 158, no. 4, pp. 452-471, 2007.

[10] A. Tarski, A. Mostowski, and R. Robinson, "Undecidable theories," North-Holland, 1953.

[11] J. Wang and J. Hao, "A new version of 2-tuple fuzzy linguistic representation model for computing with words," IEEE Trans. Fuzzy Syst., vol. 14, no. 3, pp. 435-445, 2006.

[12] J.-H. Wang and J. Hao, "An approach to computing with words based on canonical characteristic values of linguistic labels," IEEE Trans. Fuzzy Syst., vol. 15, no. 4, pp. 593-604, 2007.

Received on July 19, 2017

Revised on November 09, 2017 